\section{Periodic Structure in Ice}

A CONCAVE metal vessel, $5 \mathrm{~cm} . \times 3.75 \mathrm{~cm} . \times 1 \cdot 2$ $\mathrm{cm}$. at its deepest point, resting in natural contact (that is, at greatest convexity of its convex surface) with a stone pavement, became filled with rain water or melted sleet. It remained overnight, and held next morning a plano-convex lens of ice, which exhibited a beautiful periodic structure.

The periodic structure consisted of about a dozen very distinct colourless concentric circles, $0 \cdot 5-1 \mathrm{~mm}$. radial variation, the least, central, one about $1 \mathrm{~cm}$. diameter, with transparent ice between the rings. The structure persisted through the depth of the lens, the largest circle being, of course, of least depth. A small piece of black foreign matter (perhaps a smut) which may, or may not, have had something to do with the formation of the structure, was frozen into the centre of the smallest circle.

In reporting a previous observation of periodic structures in carbon films due to oil drops ${ }^{1}$, the absence of colloids, chemical action and dissolution was stressed. But in this present observation, which also is probably original, one chemical substance alone appears to be concerned. There is, of course, the possibility of 'heavy' water and of two phases - solid and liquid-being concerned, though the ring structures seemed as solid as the clear ice. The original liquid might also have contained a slight amount of dissolved impurity, if only condensation nuclei. Attempts will be made, artificially, to reproduce such periodic structures in ice.

\section{Denton Avenue,}

S. C. Buacktin.

Leeds, 8.

March 15.

NATURE, 129, 401, March 12, 1932.

\section{Loss of Mass in Binary Systems}

Some years ago, Sir James Jeans ${ }^{1}$ considered the problem of the variation in the orbital elements of a binary star in which one or both of the components is losing mass by radiation. He concluded that, in these circumstances, the orbital eccentricity will remain constant, whereas the semi-axis major varies inversely as the sum of the masses of the components.

Shortly after, Prof. E. W. Brown ${ }^{2}$ discussed the same problem from a different point of view, arriving at the conclusion that the orbital eccentricity varies inversely as the sum of the masses, while the semiaxis increases at a still higher rate. His paper was criticised by Jeans ${ }^{3}$, but the cause of their fundamental disagreement was not then, nor has since, been cleared up.

An investigation which has recently been completed throws light on this difficulty. Starting from the equations of motion in Cartesian form as given by Jeans, the differential equation of the orbit is deduced, and its general solution obtained. It is found that Jeans's result is justified, and the source of Brown's error is explained.

The main result of the investigation is that the loss of mass through radiation leads to the relation : semi-axis major is inversely proportional to the mass of the system, which holds throughout the life of the binary; or alternatively,

$$
P / a^{2}=\text { constant, }
$$

that is, $\log P-2 \log a^{\prime \prime}+2 \log \pi^{\prime \prime}=$ constant in the usual notation.

A statistical study of all the available material for the visual binaries has shown that not only does this relation hold throughout the life of any one star, but also it holds statistically at the present epoch for all visual binaries in the form

$\log P-2 \log a^{\prime \prime}+2 \log \pi^{\prime \prime}=-0.826 \pm 0.098$.

This result shows that all such binaries apparently originate with nearly equal values of the quantity $P / a^{2}$, the degree of scatter of individual values from the mean being given by the probable error 0.098 of the constant 0.826 .

This relation leads to a form of hypothetical parallax which we may call the mass-radiation parallax, and which has been computed for 123 binaries the orbital data of which can be taken as well determined. The computed values are found to agree very well with those found on the basis of other methods.

The above relation does not appear to hold for the eclipsing or the short period spectroscopic binaries The reason for this difference between the short and the long period binaries is probably bound up with the difference in origin of these two groups, but as yet no adequate explanation of the observations has been forthcoming.

University of the Witwatersrand,

A. E. H. Bleksley.

Johannesburg,

South Africa.

Feb. 18.

${ }^{1}$ Mon. Not. Roy. Ast. Soc., 85, 2 ; 1924

s Mon. Not. Roy. Ast. Soc., 85, 912; 1925.

Calcium Isotope with Mass $4 \mathrm{I}$ and the Radioactive Half-period of Potassium

THE values given in a previous note ${ }^{1}$ for the radioactive half-period of the potassium isotope with mass 41 , as derived from the abnormality in the atomic weight of calcium extracted from Rhiconich and Portsoy pegmatites, stand in need of correction.

In the first place, it has been learned through the kindness of Prof. Arthur Holmes, of the University of Durham, to whom a sample of the Rhiconich rock had been sent, that the Geological Survey analysis of this pegmatite ${ }^{2}$ was not applicable to the material actually used, which had not been hand-picked. Independent analysis conducted by Dr. Winifred Guthrie in this laboratory and by Dr. A. W. Groves of the Royal College of Science agreed in assigning to this material a $\mathrm{CaO}$ content of 0.55 per cent (instead of $0 \cdot 27$ ) and $\mathrm{K}_{2} \mathrm{O}$ content of $8 \cdot 0$ per cent (instead of $9 \cdot 35$ ).

The Portsoy pegmatite, which had been handpicked, gave analyses much closer to the Geological Survey figures, namely, $\mathrm{CaO} 0.28$ per cent (exact agreement) and $\mathrm{K}_{2} \mathrm{O} 8.0$ per cent (instead of $8 \cdot 9$ ). The age of 600 million years assumed for this rock in our previous note, however, is decidedly higher than geological evidence warrants, and a value of 400 million years may be regarded as more reasonable.

While the necessary recalculations were being made in the light of the above changes, it transpired that an unsuspected constant error had entered into our earlier calculations and that the life-periods arrived at were accordingly all too high. The final values for the half-period of the potassium isotope with mass 41 now obtained are : 